\title{
Reliability and validity of the Depression and Somatic Symptoms Scale among patients with chronic low back pain
}

This article was published in the following Dove Medical Press journal: Neuropsychiatric Disease and Treatment

\author{
Chun-Hao Liu',2,* \\ Tsai-Sheng $\mathrm{Fu}^{2,3, *}$ \\ Chin-Pang Lee ${ }^{1,2}$ \\ Ching-I Hung ${ }^{1,2}$ \\ 'Department of Psychiatry, Chang \\ Gung Memorial Hospital, Linkou, \\ Taiwan; ${ }^{2}$ Chang Gung University, \\ Taoyuan, Taiwan; ${ }^{3}$ Department of \\ Orthopedics, Chang Gung Memorial \\ Hospital, Keelung, Taiwan \\ *These authors contributed equally \\ to this work
}

Background: The Depression and Somatic Symptoms Scale (DSSS), which is a free scale that includes a depression subscale (DS) and a somatic subscale (SS), was developed to evaluate depression and somatic symptoms simultaneously. This study aimed to examine the reliability and validity of the DSSS among patients with chronic low back pain (CLBP).

Methods: Two-hundred and twenty-five patients with CLBP were enrolled. Psychiatric diagnoses were made based on the Structured Clinical Interview for Diagnostic and Statistical Manual of Mental Disorders-IV-Text Revision. The DSSS, Oswestry Disability Index, Hospital Anxiety and Depression Scale (HADS), and Short Form 36 (SF-36) were administered. Cronbach's alpha was used to test internal consistency. Receiver operating characteristic (ROC) analysis was used to identify cutoff scores for a major depressive episode (MDE).

Results: Subjects with an MDE $(\mathrm{N}=21)$ had greater severities of depression, anxiety, somatic symptoms, and disability as compared with those without an MDE. The Cronbach's alpha values of the DS and SS were 0.90 and 0.83 , respectively. The DS and SS were significantly correlated with the Oswestry Disability Index, the HADS, and the SF-36 subscales. The DS had the greatest area under the receiver operating characteristic curve (0.96) as compared with the SS and the HADS subscales. The cutoff score for an MDE was a DS score $\geq 15$ (sensitivity and specificity: $100 \%$ and $88.7 \%$, respectively).

Conclusion: The DSSS subscales were of acceptable reliability and validity. The DS can be used as a tool for evaluating the severity of depression and detecting an MDE in patients with CLBP. Keywords: back pain, cutoff point, depression, psychometrics, somatization

\section{Introduction}

Chronic low back pain (CLBP) is a common symptom, rather than a disease, in countries worldwide. ${ }^{1}$ Low back pain was found to have a prevalence of $11.9 \%$ and a 1 -month prevalence of $23.2 \%$ in a globalized systemic review, affecting most commonly middle-aged to elderly females. ${ }^{2}$ In the Global Burden of Disease 2010 Study, low back pain ranked the highest among other conditions in terms of years lived with disability, and ranked the sixth reason for disability-adjusted life years. ${ }^{3}$ As a result, CLBP attracts significant costs worldwide, estimated at an indirect cost of around US\$18.5 billion to US\$28.2 billion in the US ${ }^{4}$ and AUS\$9 billion in Australia. ${ }^{5}$

The strong association between CLBP and depressive disorders is well-known. CLBP is a common physical complaint among patients with major depressive disorder (MDD) and is related to morbidity. ${ }^{6}$ On the other hand, many studies have suggested that CLBP is related to psychological distress, especially anxiety, depression, and somatization, ${ }^{7-9}$ among which depression ${ }^{10}$ and somatization ${ }^{11}$ have been identified as
Department of Psychiatry, Chang Gung Memorial Hospital, Linkou, No 5, Fusing Street, Kwei-Shan, Taoyuan, Taiwan Tel +88633281200 ext 2439 Fax +88633280267 Email a36250@cloud.cgmh.org.tw 
factors associated with a poor outcome. Several psychometric scales have been used in patients with low back pain, such as the Short Form 36 (SF-36) ${ }^{12,13}$ and the Hospital Anxiety and Depression Scale (HADS); ${ }^{14,15}$ however, most psychometric scales fail to measure depression and somatic symptoms simultaneously.

As somatic symptoms are common among patients with depression, the Depression and Somatic Symptoms Scale (DSSS) was designed as a free, self-assessment scale that simultaneously evaluates depression and somatic symptoms. ${ }^{16}$ Previous studies have demonstrated that the psychometric properties of the DSSS are appropriate for use in patients with MDD, ${ }^{17-19}$ migraine, ${ }^{20,21}$ and online gaming addiction, ${ }^{22}$ and in patients under the care of a psychiatric service. ${ }^{23,24}$ However, the psychometric properties of the DSSS have not been established in patients with CLBP. Therefore, the aim of this study was to examine the reliability and validity of the DSSS in patients with CLBP.

\section{Methods}

\section{Subjects}

This study was conducted in the orthopedic clinic of a medical center in northern Taiwan, from August 2008 to November 2010. Patients were included if they were: 1) aged between 20 and 65 years; 2) were making a first visit to our orthopedics clinic; and 3) had suffered low back pain for more than 3 months. Patients were excluded from the study if they had taken antipsychotics or antidepressants within the past 4 weeks or had difficulty being interviewed.

\section{Ethical considerations}

This study was approved by the Institutional Review Board of Chang Gung Memorial Hospital. The study procedures were explained in detail to all participants based on the guidelines regulated in the Declaration of Helsinki prior to written informed consent being obtained.

\section{Diagnosis of MDD}

A psychiatrist, who was blind to the CLBP-related data, interviewed all the participants. MDD and a major depressive episode (MDE) were diagnosed based on the Structured Clinical Interview for Diagnostic and Statistical Manual of Mental Disorders-IV-Text Revision (SCID-IV-TR). ${ }^{25}$

\section{Depression and Somatic Symptoms Scale}

The DSSS is composed of a 12-item depression subscale (DS) and a ten-item somatic subscale (SS), which includes a five-item pain subscale (PS). ${ }^{17}$ Each item is scored from 0 to 3 (absent, mild, moderate, and severe) according to the severity of symptoms, with the total score ranging from 0 to 36 and 0-30 for the DS and the SS, respectively. The DSSS is significantly correlated with the Hamilton Depression Rating Scale in patients with MDD, ${ }^{26}$ and has been validated for use in patients with MDD in Taiwan ${ }^{27}$ and the US. ${ }^{28}$

\section{Oswestry Disability Index (ODI)}

The ODI is a ten-item scale used to evaluate back or leg pain-related disability in daily life. ${ }^{29,30}$ The total score ranges from 0 to 50 , and is usually multiplied by 2 to become a percentage score, indicating severe disability if the percentage score is $\geq 40$. This is one of the most commonly used scales for evaluating disability due to low back pain.

\section{Short Form 36 (SF-36)}

The SF-36, which measures health-related quality of life (HRQoL), surveys general psychological well-being, with higher scores representing better health. ${ }^{31}$ It is composed of eight domains: physical functioning, role limitations-physical, bodily pain, general health perceptions, vitality, social functioning, role limitations-emotional, and mental health. Each domain score ranges from 0 (lowest well-being) to 100 (highest well-being). ${ }^{32}$ An acute version of the SF-36, which evaluates HRQoL in the past week, was used in this study to ensure that the evaluation duration was compatible with those of the other psychometric scales employed.

\section{Hospital Anxiety and Depression Scale}

The HADS is commonly used in general medical patients and psychiatric patients. The HADS is a self-assessment scale with a seven-item anxiety subscale (HADS-A) and a sevenitem depression subscale (HADS-D). ${ }^{33}$ Each item is scored from 0 to 3 , with a total score ranging from 0 to 21 for each subscale. Higher scores indicate more severe distress, and the total score is categorized as follows: $0-7$, normal; 8-10, mild; 11-14, moderate; and 15-21, severe.

\section{Statistical methods}

The Mann-Whitney $U$ test and the chi-squared test were used to assess differences in demographic data and psychometric scores between the MDE group and the non-MDE group. Cronbach's alpha was used to test the internal consistency of the DSSS. Furthermore, the associations between the DSSS subscales and other scales (HADS subscales, SF-36, and ODI) were examined using Pearson correlations. A $P$-value $<0.05$ was determined to be significant in this study. Receiver operating characteristic (ROC) analysis was used to determine the cutoff points for a current MDE 
on the subscales of the DSSS. The area under the ROC curve (AUC) was calculated, and ranged from 0.5 to 1 , a greater AUC indicating a better ability to differentiate the two groups. The sensitivity, specificity, positive predictive value, and negative predictive value of each threshold score were also calculated. All statistical analyses were performed using SPSS 17.0 for Windows (SPSS Inc., Chicago, IL, USA).

\section{Results \\ Subjects}

Among 243 patients (133 males) who fulfilled the criteria for this study, 225 (122 males) agreed to participate in the study. The demographic data are presented in Table 1. Compared with the non-MDE group ( $n=204)$, the MDE group $(n=21)$ contained significantly more female patients, subjects with a longer duration of CLBP, and patients with greater painrelated disability.

\section{Internal consistency and reliability}

The Cronbach's alpha values of the DS, SS, and DSSS were $0.90,0.83$, and 0.93 , respectively.

\section{Distinct-groups validity}

Compared with patients in the non-MDE group, patients in the MDE group had significantly higher scores on all subscales of the DSSS and HADS, and significantly lower scores on all subscales of the SF-36, with the exception of the physical functioning subscale.

\section{Convergent validity}

The DS and SS were significantly correlated with the subscales of the HADS in patients with CLBP (Table 2). Both the DSSS and HADS subscales were significantly and negatively correlated with all domains of the SF-36 and positively correlated with the ODI (Table 2).

\section{Cut-off scores}

We found that the DS had the greatest AUC (0.962), followed by the SS (0.892) (Table 3), HADS-A (0.881), and HADS-D (0.860) subscales (not shown). At a cutoff score of $\geq 15$, the DS showed the best diagnostic quality, with $100 \%$ sensitivity and $88.7 \%$ specificity for the detection of an MDE.

\section{Discussion}

This study aimed to examine the reliability and validity of the DSSS among patients with CLBP. The results showed that 1) the DSSS was of good internal consistency and reliability; 2) the scores of the DS and SS differed significantly between patients with and without an MDE (distinct-groups validity); 3 ) the DS and SS were significantly correlated with the ODI, all subscales of the SF-36, the HADS-D, and the HADS-A (convergent validity); 4) the DS was of good sensitivity and

Table I Demographic variables and psychometric scores (mean \pm SD) in patients with and without a major depressive episode

\begin{tabular}{|c|c|c|c|c|}
\hline Parameters & $\begin{array}{l}\text { Whole sample } \\
(\mathrm{n}=225)\end{array}$ & $\begin{array}{l}\text { MDE } \\
(n=21)\end{array}$ & $\begin{array}{l}\text { Non-MDE } \\
(n=204)\end{array}$ & $P$-value \\
\hline Age (years) & $40.7 \pm I I .4$ & $40.1 \pm 10.6$ & $40.7 \pm I I .5$ & 0.82 \\
\hline Gender (\% male) & $54.2 \%$ & $33.3 \%$ & $56.4 \%$ & 0.04 \\
\hline Married & $69.3 \%$ & $57.1 \%$ & $70.6 \%$ & 0.20 \\
\hline Employed & $67.6 \%$ & $57.1 \%$ & $68.6 \%$ & 0.28 \\
\hline Total duration of LBP (months) & $60.2 \pm 81.8$ & $98.3 \pm 87.7$ & $56.3 \pm 80.4$ & 0.003 \\
\hline Pain frequency (days per month) & $19.8 \pm 10$ & $23.4 \pm 9.0$ & $19.5 \pm 10.1$ & 0.137 \\
\hline ODI & $31.4 \pm 15.3$ & $39.3 \pm 14.4$ & $30.6 \pm 15.2$ & 0.011 \\
\hline DS & $8.9 \pm 7.1$ & $21.7 \pm 5.3$ & $7.6 \pm 5.8$ & $<0.001$ \\
\hline SS & $8.7 \pm 5$ & $16.1 \pm 4.7$ & $7.9 \pm 4.4$ & $<0.001$ \\
\hline HADS-D & $5.8 \pm 4.1$ & 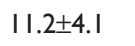 & $5.3 \pm 3.7$ & $<0.001$ \\
\hline HADS-A & $7.3 \pm 4.4$ & $13.0 \pm 3.1$ & $6.7 \pm 4.1$ & $<0.001$ \\
\hline $\mathrm{PF}$ & $67.8 \pm 20.8$ & $60.2 \pm 20.6$ & $68.6 \pm 20.7$ & 0.067 \\
\hline RP & $31 \pm 37.9$ & $8.3 \pm 24.2$ & $33.3 \pm 38.3$ & 0.001 \\
\hline $\mathrm{BP}$ & $46.2 \pm 16.9$ & $32.0 \pm 10.9$ & $47.6 \pm 16.7$ & $<0.001$ \\
\hline $\mathrm{GH}$ & $47.9 \pm 22.4$ & $32.2 \pm 15.9$ & $49.5 \pm 22.4$ & $<0.001$ \\
\hline VT & $50.6 \pm 23.9$ & $16.7 \pm 12.5$ & $54.1 \pm 22.0$ & $<0.001$ \\
\hline SF & $68.4 \pm 24.2$ & $40.5 \pm 22.7$ & $71.3 \pm 22.5$ & $<0.001$ \\
\hline $\mathrm{RE}$ & $53.8 \pm 43.8$ & $4.8 \pm 15.9$ & $58.8 \pm 42.7$ & $<0.001$ \\
\hline $\mathrm{MH}$ & $61.3 \pm 22.1$ & $29.0 \pm 13.7$ & $64.6 \pm 20.1$ & $<0.001$ \\
\hline
\end{tabular}

Abbreviations: BP, bodily pain subscale; DS, depression subscale of the DSSS; DSSS, Depression and Somatic Symptoms Scale; GH, general health perceptions subscale; HADS-A, anxiety subscale of the HADS; HADS-D, depression subscale of the HADS; HADS, Hospital Anxiety and Depression Scale; LBP, low back pain; MDE, major depressive episode; $\mathrm{MH}$, mental health subscale; ODI, Oswestry Disability Index; PF, physical functioning subscale; RE, role limitations-emotional subscale; RP, role limitationsphysical subscale; SF, social functioning subscale; SS, somatic subscale of the DSSS; VT, vitality subscale. 
Table 2 Pearson correlation coefficients of the subscales of the DSSS and HADS with the subscales of the Short Form 36 and the Oswestry Disability Index

\begin{tabular}{l|l|l|l|l}
\hline Subscales & DS & SS & HADS-A & HADS-D \\
\hline PF & $-0.33^{*}$ & $-0.26^{*}$ & $-0.30^{*}$ & $-0.39^{*}$ \\
RP & $-0.36^{*}$ & $-0.22^{*}$ & $-0.32^{*}$ & $-0.40^{*}$ \\
BP & $-0.43^{*}$ & $-0.42^{*}$ & $-0.34^{*}$ & $-0.31^{*}$ \\
GH & $-0.50^{*}$ & $-0.42^{*}$ & $-0.42^{*}$ & $-0.43^{*}$ \\
VT & $-0.72^{*}$ & $-0.52^{*}$ & $-0.65^{*}$ & $-0.69^{*}$ \\
SF & $-0.67^{*}$ & $-0.50^{*}$ & $-0.55^{*}$ & $-0.64^{*}$ \\
RE & $-0.58^{*}$ & $-0.42^{*}$ & $-0.4 I^{*}$ & $-0.50^{*}$ \\
MH & $-0.78^{*}$ & $-0.5 I^{*}$ & $-0.76^{*}$ & $-0.69^{*}$ \\
ODI & $0.4 I^{*}$ & $0.29^{*}$ & $0.32^{*}$ & $0.46^{*}$ \\
HADS-A & $0.76^{*}$ & $0.53^{*}$ & N/A & N/A \\
HADS-D & $0.74^{*}$ & $0.45^{*}$ & N/A & N/A \\
\hline
\end{tabular}

Note: $* p<0.01$

Abbreviations: BP, bodily pain subscale, DS, depression subscale of the DSSS; DSSS, Depression and Somatic Symptoms Scale; GH, general health perceptions subscale; HADS-A, anxiety subscale of the HADS; HADS-D, depression subscale of the HADS; HADS, Hospital Anxiety and Depression Scale; $\mathrm{MH}$, mental health subscale; N/A, not applicable; ODI, Oswestry Disability Index; PF, physical functioning subscale; RE, role limitations-emotional subscale; RP, role limitations-physical subscale; SF, social functioning subscale; SS, somatic subscale of the DSSS; VT, vitality subscale.

specificity for the detection of an MDE. These results demonstrated that the DSSS had a good reliability and validity for use in patients with CLBP. Moreover, the DS could be used as a tool to screen for an MDE.

Several points were worthy of note: 1) The DS and SS were highly-correlated with the ODI. This demonstrated that the two subscales could reflect the severity of disability due to CLBP. 2) This study found that the DS had significantly moderate to high correlations with the SF-36 and HADS. The SF-36 focuses on general psychological well-being, and has a sensitivity of $80 \%$ and specificity of $90 \%$ in detecting depressive symptoms among patients with chronic spinal pain. ${ }^{13}$ The HADS has been validated for use in patients with acute low back pain. ${ }^{15}$ However, it has been criticized due to conceptual issues and the combination of the two related factors of anxiety and depression..$^{34} 3$ ) The DS had the greatest AUC as compared with the SS, the HADS-A, and the HADS-D. This demonstrated that the DS had a better diagnostic property than the other subscales for the screening of an MDE among patients with CLBP. 4) Somatic complaints in patients with chronic pain easily lead to a falsepositive or over-diagnosis of depression. ${ }^{35}$ Therefore, the DS is more accurate and appropriate for the detection of an MDE as compared with the SS and the full DSSS.

The HADS and the DSSS are self-assessment scales. The role of a self-assessment scale is not to serve as a diagnostic tool, but instead as a screening tool to raise the clinician's awareness and to initiate further diagnostic evaluation. ${ }^{13}$ The 12-item DS has a lower respondent burden in the clinical setting than the 14-item HADS or the 36-item SF-36. The DSSS has other advantages of use in patients with CLBP: first, the DSSS can evaluate depression and somatic symptoms simultaneously; second, it is a free scale; and third, the DS was designed based on the criteria of an MDE. ${ }^{17}$ Therefore, the severity of depression as evaluated by the DS should be more compatible with an MDE in comparison with the HADS-D and the HADS-A. This might be the reason for which the AUC of the DS was greater than that of the HADS-D. This result demonstrated that the DS might be a good choice for MDE screening in patients with CLBP.

\section{Limitations}

This study had some limitations. First, the study was performed in the orthopedic clinic of a medical center. The characteristics of the patients with CLBP might differ in different clinical settings, and expansion of the results to other settings should be performed cautiously. Second, this study set several inclusion and exclusion criteria, which may have led to bias during the enrollment process. We did not collect the demographic data from those who refused to participate in our study, and the responsiveness was not recorded. Third, there are many methods by which to validate a psychometric scale, of which this study only used a limited number to test the validity of the DSSS in patients with CLBP. We have done factor analysis in our previous study, and it showed that most of items in DS and SS subscales belong to the depression

Table 3 Sensitivity, specificity, PPV, and NPV at different cutoff scores for a major depressive episode in patients with chronic low back pain

\begin{tabular}{l|l|l|l|l|l|l}
\hline Subscales & Score & Sensitivity (\%) & Specificity (\%) & PPV (\%) & NPV (\%) & AUC \\
\hline DS & $\geq 14$ & 1 & 0.863 & 0.880 & 1.000 & \\
& $\geq 15$ & 1 & 0.887 & 0.898 & 1.000 & 0.962 \\
SS & $\geq 16$ & 0.857 & 0.926 & 0.921 & 0.866 & \\
& $\geq 11$ & 0.81 & 0.624 & 0.683 & 0.767 & \\
& $\geq 12$ & 0.81 & 0.838 & 0.833 & 0.815 & 0.892 \\
& $\geq 13$ & 0.762 & 0.877 & 0.861 & 0.787 & \\
\hline
\end{tabular}

Abbreviations: AUC, area under curve; DS, depression subscale of the DSSS; DSSS, Depression and Somatic Symptoms Scale; NPV, negative predictive value; PPV, positive predictive value; SS, somatic subscale of the DSSS. 
and somatic factors. ${ }^{16}$ But other methods, such as test-retest reliability, to test the validity of the DSSS are indicated in future studies. Finally, there was only one psychiatrist who made all the diagnoses, and the accuracy had been questioned despite the diagnosis was made based on the SCID-IV.

\section{Conclusion}

This study demonstrated that the DSSS had good psychometric properties among patients with CLBP, especially the DS. The two subscales of the DSSS were significantly correlated with depression, anxiety, psychological well-being, and disability in patients with CLBP. Owing to its brevity and high sensitivity, the DS can be used as a screening tool for the detection of an MDE in patients with CLBP. In future studies, other methods should be used to test the validity of the DSSS.

\section{Announcement}

Our team agrees that physicians, researchers, and pain-related workers may freely use this scale in clinical practice and research. We hope that the DSSS can help physicians and patients to become more aware of the interaction of mental and physical symptoms in depression.

\section{Availability of data and materials}

The datasets used and/or analyzed during the current study are available from the corresponding author on reasonable request.

\section{Acknowledgments}

The authors are thankful for the statistical assistance and acknowledge the support of the Maintenance Project of the Center for Big Data Analytics and Statistics (Grant CLRPG3D0045) at Chang Gung Memorial. This study was supported in part by the Chang Gung Memorial Hospital Research Program (CMRPG 371701) and National Science Council grants (NSC 102-2314-B-182A007).

\section{Author contributions}

$\mathrm{CIH}$ and TSF designed and conducted the study. CHL and CPL analyzed the data. CHL drafted the manuscript. All authors contributed to data analysis, drafting or revising the article, gave final approval of the version to be published, and agree to be accountable for all aspects of the work.

\section{Disclosure}

The authors report no conflicts of interest in this work.

\section{References}

1. Maher C, Underwood M, Buchbinder R. Non-specific low back pain. Lancet. 2017;389(10070):736-747.
2. Hoy D, Bain C, Williams G, et al. A systematic review of the global prevalence of low back pain. Arthritis Rheum. 2012;64(6):2028-2037.

3. Hoy D, March L, Brooks P, et al. The global burden of low back pain: estimates from the Global Burden of Disease 2010 study. Ann Rheum Dis. 2014;73(6):968-974.

4. Dagenais S, Caro J, Haldeman S. A systematic review of low back pain cost of illness studies in the United States and internationally. Spine J. 2008;8(1):8-20.

5. Walker BF, Muller R, Grant WD. Low back pain in Australian adults: the economic burden. Asia Pac J Public Health. 2003;15(2):79-87.

6. Ohayon MM, Schatzberg AF. Using chronic pain to predict depressive morbidity in the general population. Arch Gen Psychiatry. 2003; 60(1):39-47.

7. Bener A, Verjee M, Dafeeah EE, et al. Psychological factors: anxiety, depression, and somatization symptoms in low back pain patients. J Pain Res. 2013;6:95-101.

8. Gatchel RJ, Peng YB, Peters ML, Fuchs PN, Turk DC. The biopsychosocial approach to chronic pain: scientific advances and future directions. Psychol Bull. 2007;133(4):581-624.

9. Bener A, El-Rufaie OF, Kamran S, Georgievski AB, Farooq A, Rysavy M. Disability, depression and somatization in a low back pain population. Int J Rheum Dis. 2006;9(3):257-263.

10. Pinheiro MB, Ferreira ML, Refshauge K, et al. Symptoms of depression as a prognostic factor for low back pain: a systematic review. Spine J. 2016;16(1):105-116.

11. Ailliet L, Rubinstein SM, Knol D, van Tulder MW, de Vet HC. Somatization is associated with worse outcome in a chiropractic patient population with neck pain and low back pain. Man Ther. 2016;21:170-176.

12. Ferrari R. Responsiveness of the short-form 36 and oswestry disability questionnaire in chronic nonspecific low back and lower limb pain treated with customized foot orthotics. J Manipulative Physiol Ther. 2007;30(6):456-458.

13. Walsh TL, Homa K, Hanscom B, Lurie J, Sepulveda MG, Abdu W. Screening for depressive symptoms in patients with chronic spinal pain using the SF-36 Health Survey. Spine J. 2006;6(3):316-320.

14. Snekkevik H, Eriksen HR, Tangen T, Chalder T, Reme SE. Fatigue and depression in sick-listed chronic low back pain patients. Pain Med. 2014;15(7):1163-1170.

15. Turk DC, Dworkin RH, Trudeau JJ, et al. Validation of the hospital anxiety and depression scale in patients with acute low back pain. J Pain. 2015;16(10):1012-1021.

16. Hung CI, Weng LJ, Su YJ, Liu CY. Depression and somatic symptoms scale: a new scale with both depression and somatic symptoms emphasized. Psychiatry Clin Neurosci. 2006;60(6):700-708.

17. Hung CI, Weng LJ, Su YJ, Liu CY. Preliminary study of a scale measuring depression and somatic symptoms. Psychol Rep. 2006;99(2): 379-389.

18. Hung CI, Wang SJ, Liu CY. Validation of the depression and somatic symptoms scale by comparison with the Short Form 36 scale among psychiatric outpatients with major depressive disorder. Depress Anxiety. 2009;26(6):583-591.

19. Woo JM, Jeon HJ, Noh E, et al. Importance of remission and residual somatic symptoms in health-related quality of life among outpatients with major depressive disorder: a cross-sectional study. Health Qual Life Outcomes. 2014;12:188.

20. Hung CI, Liu CY, Cheng YT, Wang SJ. Migraine: a missing link between somatic symptoms and major depressive disorder. $J$ Affect Disord. 2009;117(1-2):108-115.

21. Hung CI, Liu CY, Yang CH, Wang SJ. The impacts of migraine among outpatients with major depressive disorder at a two-year follow-up. PLoS One. 2015;10(5):e0128087.

22. Wei HT, Chen MH, Huang PC, Bai YM. The association between online gaming, social phobia, and depression: an internet survey. $B M C$ Psychiatry. 2012;12:92.

23. Chang CC, Wu TH, Chen CY, Wang JD, Lin CY. Psychometric evaluation of the internalized stigma of mental illness scale for patients with mental illnesses: measurement invariance across time. PLoS One. 2014;9(6):e98767. 
24. Wu TH, Chang CC, Chen CY, Wang JD, Lin CY. Further psychometric evaluation of the self-stigma scale-short: measurement invariance across mental illness and gender. PLoS One. 2015;10(2):e 0117592.

25. First MBSR, Gibbon M, Williams JBW. Structured Clinical Interview for DSM-IV-TR Axis I Disorders, Research Version, Patient Edition $(S C I D-I / P)$. New York, NY: Biometrics Research, New York State Psychiatric Institute; 2002.

26. Hung CI, Liu CY, Wang SJ, Juang YY, Yang CH. Somatic symptoms: an important index in predicting the outcome of depression at six-month and two-year follow-up points among outpatients with major depressive disorder. J Affect Disord. 2010;125(1-3):134-140.

27. Hung CI, Liu CY, Wang SJ, Yao YC, Yang CH. The cut-off points of the depression and somatic symptoms scale and the hospital anxiety and depression scale in detecting non-full remission and a current major depressive episode. Int J Psychiatry Clin Pract. 2012;16(1):33-40.

28. Tse PS, González DA, Jenkins SR. Validating the structure of the depression and somatic symptoms scale. Psychosomatics. 2018;59(3): $277-282$.
29. Chapman JR, Norvell DC, Hermsmeyer JT, et al. Evaluating common outcomes for measuring treatment success for chronic low back pain. Spine. 2011;36(21 Suppl):S54-S68.

30. Fairbank JC, Couper J, Davies JB, O’Brien JP. The Oswestry low back pain disability questionnaire. Physiotherapy. 1980;66(8):271-273.

31. Ware JKM, Keller S. SF-36 Physical and Mental Health Summary Scale: A User's Manual. Boston: Health Assessment Lab; 1994.

32. Brazier JE, Harper R, Jones NM, et al. Validating the SF-36 health survey questionnaire: new outcome measure for primary care. BMJ. 1992; 305(6846):160-164.

33. Zigmond AS, Snaith RP. The hospital anxiety and depression scale. Acta Psychiatr Scand. 1983;67(6):361-370.

34. Coyne JC, van Sonderen E. No further research needed: abandoning the Hospital and Anxiety Depression Scale (HADS). J Psychosom Res. 2012;72(3):173-174.

35. Turk DC, Okifuji A. Detecting depression in chronic pain patients: adequacy of self-reports. Behav Res Ther. 1994;32(1):9-16.

\section{Publish your work in this journal}

Neuropsychiatric Disease and Treatment is an international, peerreviewed journal of clinical therapeutics and pharmacology focusing on concise rapid reporting of clinical or pre-clinical studies on a range of neuropsychiatric and neurological disorders. This journal is indexed on PubMed Central, the 'PsycINFO' database and CAS, and is the official journal of The International Neuropsychiatric Association (INA). The manuscript management system is completely online and includes a very quick and fair peer-review system, which is all easy to use. Visit http://www.dovepress.com/testimonials.php to read real quotes from published authors. 